\title{
A IMPORTÂNCIA DA PREVISÃO DO TEMPO NA PREVENÇÃO DE RISCOS METEOROLÓGICOS
}

JoÃo CORTE-REAL ${ }^{1}$

\begin{abstract}
Resumo - Este artigo trata do fundamento científico da previsão do tempo, bem como de aspectos operacionais fundamentais a ela associados. Em particular mostra-se como o início da moderna previsão do tempo resultou da visão de John von Neumann, que interligou de forma brilhante ciência, tecnologia e gestão.
\end{abstract}

Palavras-chave: Espaço, meteorologia, risco, modelos numéricos.

Abstract - THE RELEVANCE OF WEATHER FORECASTING IN THE PREVENTION OF METEOROLOGICAL HAZARDS. This paper discusses the scientific foundations of weather prediction, as well as the fundamental operational aspects associated with weather forecasting. The paper attributes the beginning of modern numerical weather prediction to the vision of John von Neumann, who brilliantly connected science, technology and management.

Keywords: Space, meteorology, hazard, risk, numerical models.

\section{INTRODUÇÃO}

Nos últimos 50 anos, tempo de vida da Finisterra, as ciências da atmosfera conheceram um impulso sem precedentes. Tal circunstância deve-se à forte interacção, não linear, entre conhecimento científico e desenvolvimento tecnológico, que permitiu a concepção e construção de instrumentos de observação avançados, de computadores digitais com desempenhos crescentes, os quais tornaram possível o desenvolvimento da previsão

Recebido: Abril 2015. Aceite: Julho 2015.

1 João Alexandre Medina Corte-Real, Professor catedrático aposentado da Universidade de Évora. Colaborador do Instituto de Ciências Agrárias e Ambientais Mediterrânicas (ICAAM) da Universidade de Évora. No presente, a sua área de investigação principal centra-se na variabilidade climática e seus impactes, na modelação de cenários regionais, na hidroclimatologia e na ecohidrologia. Desde 2011, é Professor Convidado do DAT - Departamento de Aeronáutica e Transportes da Universidade Lusófona de Humanidades e Tecnologia, Lisboa. Coordenador do DREAMS (Centre for Interdisciplinary Development and Research on Environment, Applied Management and Space) da Universidade Lusófona, Lisboa, criado em 2013. E-mail : jmcr@ uevora.pt e jamcr09@gmail.com 
numérica do tempo, alargando-a à escala global com resoluções elevadas. Esta expansão do conhecimento científico e da capacidade de proteger vidas e bens, através da previsão de fenómenos atmosféricos adversos à actividade humana, ligou a meteorologia à prevenção de riscos. Neste tempo de comemorações (e de alertas permanentes) cabe pois falar do tempo, que pode representar um risco, ameaçando vidas e bens.

Neste artigo, procurar-se-á responder, numa base científica, a questões tais como: o que é o tempo? É possível prevê-lo? Se sim, como? Qual a relevância da previsão do tempo na prevenção de riscos meteorológicos e na actividade humana em geral?

\section{O CONCEITO DE ESPAÇO EM METEOROLOGIA}

A meteorologia é o estudo da atmosfera, fluido gasoso que envolve o globo. O espaço ocupado pela atmosfera é pois tridimensional e tem a potência do contínuo; é, além disso, espaço no conceito newtoniano i.e. espaço absoluto, cuja métrica é a mesma para todos os observadores. Os fenómenos atmosféricos que têm lugar nesse espaço evoluem no tempo duração (absoluto), dando assim sentido aos conceitos de análise e previsão do tempo atmosférico. Na prática, estes conceitos só podem no entanto aplicar-se a um espaço discreto, constituído por um número finito de pontos, dispostos numa rede regular e a evolução temporal é descrita apenas num conjunto discreto e finito de instantes. O conceito de espaço em meteorologia é, por consequência, distinto do conceito de espaço em geografia, embora ambos os conceitos não sejam disjuntos.

\section{A ATMOSFERA E O TEMPO}

A atmosfera é um dos componentes do sistema climático formado pela união da atmosfera, hidrosfera, criosfera, litosfera e biosfera. Este pode considerar-se um sistema fechado, mas não isolado, uma vez que recebe energia radiante do sol e perde energia radiante para o espaço. $\mathrm{O}$ sol constitui a fonte primária de energia para todos os processos que ocorrem no sistema climático. A atmosfera constitui o componente de maior variabilidade do sistema climático; trata-se de um sistema cuja circulação é turbulenta (irregular e instável), caótica (sensível às condições iniciais), no qual têm lugar processos não lineares, que ocorrem em múltiplas escalas espaço-temporais (que se influenciam mutuamente devido à não linearidade e que não podem por consequência ser analisadas independentemente umas das outras), sujeitos a mecanismos de realimentação e a teleconexões.

A atmosfera é um sistema aberto, i.e., permuta massa e energia com os restantes componentes do sistema climático em processos complexos, com as características apontadas. Diz-se que a atmosfera é forçada em múltiplas escalas espaço-temporais, pelos restantes componentes do sistema climático e pelo sol. 
A atmosfera é constituída pelo ar, mistura gasosa, e por partículas em suspensão (aerossóis). Os componentes gasosos maioritários são o oxigénio molecular (209 $500 \mathrm{ppmv}^{\mathrm{i}}$ ) e o azoto molecular (780 $800 \mathrm{ppmv}$ ); seguem-se o vapor de água (30 $000 \mathrm{ppmv}$ ) e o árgon (9 $300 \mathrm{ppmv}$ ). Os componentes minoritários, totalizando cerca de 400ppmv, incluem o dióxido de carbono, o metano, o óxido nitroso, os CFCs, entre outros, sem esquecer aerossóis.

O vapor de água e os componentes minoritários são aqueles que interactuam com a radiação, absorvendo-a ou difundindo-a, sendo portanto aqueles que determinam a distribuição espacial das regiões de aquecimento e arrefecimento da atmosfera e, consequentemente, a forma como a atmosfera se move, i.e., a circulação atmosférica.

O componente água é o único componente atmosférico que pode mudar de fase, estando quantidades apreciáveis de energia associadas às transições de fase da água; por exemplo, a condensação de $1 \mathrm{~g}$ de vapor de água, liberta $2500 \mathrm{~J} \approx 598 \mathrm{cal}$; reciprocamente a evaporação de $1 \mathrm{~g}$ de água líquida requer a absorção de 2 500J; a libertação de calor latente aquece a atmosfera e a absorção arrefece-a. Por outro lado, a uma dada pressão, a massa de água que pode existir na atmosfera na fase vapor tem um limite máximo que depende da temperatura do ar, pelo que se pode concluir que as transições de fase da água na atmosfera, e as trocas de energia associadas, são inevitáveis. A condensação do vapor de água dá lugar à formação de nuvens, as quais difundem a radiação solar e absorvem a radiação terrestre.

Num dado instante, o estado termo-hidrodinâmico da atmosfera é definido pelos valores que as concentrações dos diferentes componentes, a temperatura, a pressão, a densidade e a velocidade, tomam em cada ponto do meio. Todas estas propriedades, caracterizam a partícula do meio que, no instante referido, ocupa a posição considerada. $\mathrm{Na}$ gíria meteorológica a velocidade é designada por vento.

A concentração do vapor de água determina a humidade do ar; caso esta seja nula, diz-se que o ar é seco; na situação oposta fala-se de ar húmido.

O estado termo-hidrodinâmico da atmosfera, numa dada região, num dado instante, é o tempo nessa região, nesse instante. Podemos assim falar do tempo à superfície, às $12 \mathrm{~h}$ de hoje, em Lisboa, ou do tempo às $18 \mathrm{~h}$ de ontem, sobre o Atlântico, ou a $5500 \mathrm{~m}$ de altitude. Como o estado termo-hidrodinâmico da atmosfera se altera no decurso do tempo, o tempo atmosférico modifica-se e evolui; o tempo de hoje não é necessariamente o tempo de amanhã, ou de um mês mais tarde.

Deve notar-se que não é possível conhecer o tempo, tal como foi definido; como já foi referido, o continuum espaço-temporal é, na prática, substituído por um discretum. Esta conclusão tem importantes consequências como veremos.

\section{A PREVISÃO DO TEMPO COMO PROBLEMA CIENTÍFICO}

O que é prever o tempo? É determinar os estados futuros da atmosfera, a partir de um estado conhecido num dado instante, dito estado inicial, e de condições fronteira, que descrevem o forçamento a que a atmosfera está submetida. 
Será isto possível? Em teoria, acredita-se que sim. Porquê? Porque as variáveis que definem o estado instantâneo da atmosfera estão ligadas entre si por equações que traduzem leis fundamentais da física, que por esta razão se designam por equações primitivas, e que incluem o forçamento da atmosfera pelos restantes componentes do sistema climático e pelo sol. Por outras palavras, a evolução do tempo é determinística: conhecido um estado inicial e condições de fronteira (forçamento) os estados futuros estão determinados e podem ser conhecidos, resolvendo as equações atrás referidas. Esta ideia enfrenta no entanto obstáculos que, até ao presente, continuam a ser intransponíveis. Com efeito, sendo a circulação atmosférica caótica (caos significa neste contexto, sensibilidade às condições iniciais, i.e. o movimento é caótico se estados iniciais muito próximos, evoluírem de forma completamente distinta no decurso do tempo), um pequeno erro na definição do estado inicial vai amplificar-se no decurso do tempo; ora, como não é possível conhecer com precisão nenhum estado instantâneo, haverá sempre erros na definição do estado inicial. Esta circunstância, impõe um limite temporal à possibilidade de prever o tempo, i.e. à predictabilidade da atmosfera; assim, só será possível estender a previsão com confiança, até poucos dias subsequentes ao dia inicial. Por outro lado, o forçamento da atmosfera também não é conhecido com precisão. Finalmente, as equações de prognóstico, são equações com derivadas parciais, não lineares, acopladas, para as quais a matemática desconhece métodos de solução (com excepção de casos particulares).

Em conclusão: prever o tempo consistiria em resolver um sistema de equações, para o qual se desconhecem métodos de solução, partindo de condições iniciais e de fronteira que são imperfeitamente conhecidas. Como estas dificuldades não têm, até ao presente, sido resolvidas, o problema da previsão do tempo, tal como foi enunciado atrás, não tem solução. Por isso, a previsão do tempo, assenta em métodos aproximados, baseados em dados de observações de superfície, de altitude (radiossondagens), de satélites, de radar, e na análise numérica.

A ambição de uma solução matemática para a previsão do tempo foi colocada pelo meteorologista americano Cleveland Abbe (fig. 1) em 1890. Abbe notabilizou-se pela organização de um serviço meteorológico sistemático, por telégrafo (inventado em 1837), em benefício do comércio. Nos primeiros anos do século XX, o físico norueguês Vilhelm Bjerknes (fig. 1), da Universidade de Bergen, desenvolveu aquela ideia com o objectivo claro de, à semelhança da astronomia, converter a meteorologia numa ciência exacta, numa verdadeira física da atmosfera. Bjerknes estabeleceu duas etapas para aquilo que designou por previsão racional do tempo: a etapa de diagnóstico, em que é definido um estado inicial com base nas observações, e a etapa de prognóstico, na qual o estado inicial é projectado no futuro, num processo ditado pelas equações primitivas, deterministas. No entanto, Bjerknes reconheceu não haver qualquer possibilidade de pôr as suas ideias em prática, uma vez que nada podia fazer nem analítica nem numericamente. Em consequência, Bjerknes propôs um método gráfico para a previsão do tempo, baseado no traçado de cartas de tempo ou cartas meteorológicas, de superfície e de altitude. As cartas meteorológicas eram traçadas à mão, com base nas observações disponíveis; a comparação da carta relativa a uma dada hora, com as correspondentes a horas anteriores, per- 
mitia extrapolar, subjectivamente, as posições e intensidades dos centros de acção (depressões e anticiclones) e dos sistemas frontais, para um instante futuro. Deve notar-se que os conceitos de massa de ar, de superfície frontal e de frente, foram introduzidos pelo próprio Bjerknes e desenvolvidos pela escola de Bergen.
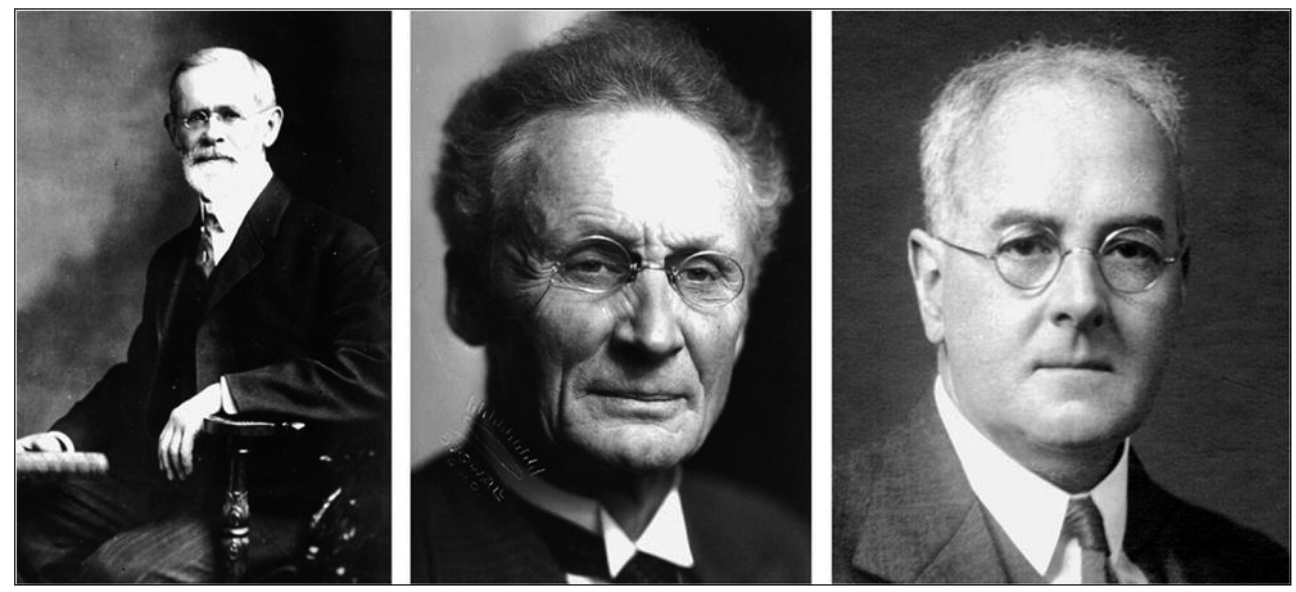

Fig. 1 - Cleveland Abbe, Vilhelm Bjerknes e Lewis Fry Richardson.

Em 1913, Lewis Fry Richardson (fig. 1), meteorologista inglês, levou a cabo a primeira tentativa para resolver as equações primitivas, utilizando calculadoras de secretária. Richardson começou por simplificar as equações do movimento, introduzindo a hipótese hidrostática e, por aplicação de diferenças finitas, obteve valores dos campos meteorológicos num instante posterior, próximo do inicial. Em seguida, iterou o processo no tempo, até cobrir um intervalo de tempo da ordem do dia. Desta forma, Richardson pôde analisar variações da pressão atmosférica e do vento em dois pontos situados na Europa central. Os resultados foram completamente desastrosos; com efeito, para só falar da pressão, os valores calculados da tendência da pressão eram da ordem de $145 \mathrm{hPa} / 6 \mathrm{~h}$, valores totalmente irrealistas, se se tiver em conta que tendências observadas são pequenas, da ordem de poucos $\mathrm{hPa}$, ou décimos de $\mathrm{hPa}$, em intervalos de tempo de $3 \mathrm{~h}$. As razões que levaram aos erros de previsão, na tentativa levada a cabo por Richardson, só foram integralmente compreendidas anos mais tarde, após desenvolvimentos teóricos cruciais, verificados na análise numérica (por Courant, Friedrichs e Lewy (Nebeker, 1995) e na dinâmica de fluidos e da atmosfera (e.g. Rossby, the "Weather Man", Nebeker, 1995), e desenvolvimentos tecnológicos verdadeiramente inovadores, designadamente a invenção da radiossonda e do computador digital, inexistentes ao tempo da tentativa de Richardson.

Mas a busca dessa compreensão foi estimulada pelo fracasso clamoroso do meteorologista inglês, que foi de facto o precursor da moderna previsão do tempo. Coube a Jule Gregory Charney (Nebeker, 1995) o mérito da realização, em 1950, da primeira previsão 
do tempo com sucesso, evitando os erros do seu predecessor. Charney havia sido convidado por John von Neumann (fig. 2), a participar no projecto, que este havia concebido e que supervisionava, de construção do primeiro computador electrónico no Institute for Advanced Study em Princeton, ao qual veio a ser dada a designação de Electronic Numerical Integrator and Computer ou ENIAC.

O ENIAC foi construído entre 1946 e 1952 e Jule Charney foi, no projecto de von Neumann, Director do Grupo 4, Meteorologia, entre 1948 e 1956. John von Neumann tinha discutido o projecto com Rossby e havia conseguido financiamento da marinha americana. Após o início do projecto, von Neumann organizou uma conferência de meteorologia, para conseguir o apoio da comunidade meteorológica, onde esteve presente Charney, que veio a assumir as funções referidas.

Em 1947 é criado em Portugal o Serviço Meteorológico Nacional (SMN), sob a direcção do Professor Herculano Amorim Ferreira ii ; em 1969, é criada, no SMN, a unidade de Previsão Matemática do Tempo, dirigida pelo Dr. Vítor Chiote Tavares.

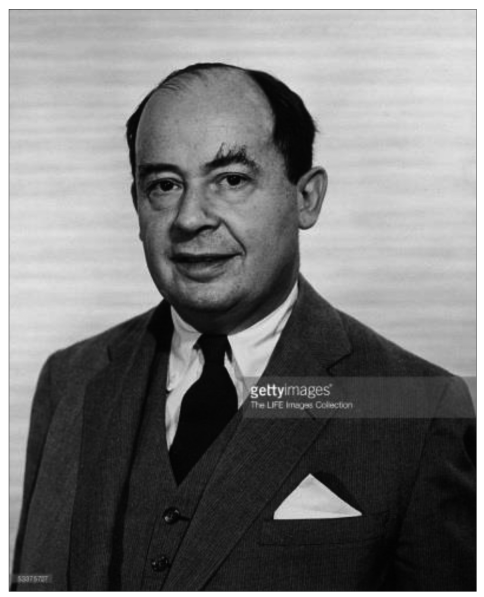

Fig. 2 - John von Neumann.

\section{A PREVISÃO OPERACIONAL DO TEMPO}

Como já referido, a previsão do tempo assenta na aplicação de métodos numéricos. Para o efeito, a atmosfera é dividida em camadas, limitadas na base e no topo por superfícies, nas quais a coordenada de posição vertical se mantém constante (e.g. superfícies de nível ou superfícies isobáricas). Cada uma daquelas superfícies, com excepção da primeira (base da primeira camada) e da última (topo da última camada), é simultaneamente topo de uma camada e base da camada seguinte. Em cada superfície, é definida uma malha, rede, ou grade "horizontal" regular, por exemplo uma malha quadrada, de dimensão $\Delta x$. Desta forma, a atmosfera fica coberta por volumes regulares (e.g. volumes 
paralelepipédicos) nos vértices dos quais, as equações primitivas são discretizadas, por aplicação de esquemas numéricos, que convertem derivadas parciais, espaciais e temporais, em diferenças finitas no espaço e no tempo. Assim, o sistema de equações primitivas fica substituído por um sistema de equações algébricas, não lineares, acopladas, para as quais há métodos de solução (aproximados), cuja aplicação depende da disponibilidade de utilização de um computador digital.

Deve notar-se que a dimensão $\Delta x$ da malha regular sobreposta a cada superfície coordenada e o tempo $\Delta t$, que define o intervalo de discretização no domínio temporal, não podem ser independentes, antes devem obedecer à condição estabelecida em 1928, por Friedrichs, Courant e Lewy (Nebeker, 1995), da Universidade de Gottingen, portanto vários anos depois da tentativa pioneira de Richardson. Do ponto de vista físico, a referida condição significa que, no intervalo de tempo $\Delta t$, a onda mais rápida, solução das equações que regem o comportamento da atmosfera, não pode percorrer uma distância igual ou superior à dimensão $\Delta x$, da malha horizontal, i.e. a condição impõe que:

\section{$\mathrm{c} \Delta t<\Delta x$}

ou

$$
\frac{c \Delta t}{\Delta x}<1
$$

onde $c$ é a velocidade de propagação da fase, da onda referida.

O não cumprimento desta condição leva, no decurso da integração numérica das equações discretizadas, ao crescimento da amplitude das correspondentes soluções; esta amplificação, sem contrapartida física, portanto espúria, representa uma instabilidade numérica de cálculo, designada por instabilidade computacional. Para valores elevados de c, o intervalo $\Delta t$ tem de ser pequeno, para um dado $\Delta x$; se as equações forem simplificadas, por forma a que ondas de alta velocidade de propagação não sejam solução, i.e. se ondas de alta velocidade de propagação forem filtradas das equações, então, para dado $\Delta x, \Delta t$ pode assumir valores mais elevados. A filtragem de soluções com velocidade de propagação elevada (ondas sonoras e gravítico-inerciais) era necessária ao tempo de Richardson e de Charney von Neumann. O problema da filtragem foi resolvido por Charney (Nebeker, 1995), o que permitiu a selecção de valores elevados para $\Delta t$ : mesmo assim, a previsão para $24 \mathrm{~h}$ durou, com o ENIAC, 1 dia!

Na tentativa de Richardson, a desconhecida condição FCL não foi observada, pois o $\Delta t$ era demasiado elevado, o que constituiu uma das causas de insucesso. Outra causa, reconhecida a posteriori, e insuspeitada por Richardson, residia nos erros contidos nos dados iniciais; esses erros comportaram-se, do ponto de vista numérico, como ondas gravítico-inerciais espúrias, de elevada amplitude e propagação rápida, responsáveis pela não observância do critério FCL. 
Como se vê, a discretização espaço-temporal da atmosfera e a aplicação de métodos numéricos têm um preço elevado e estão longe de ser intuitivas. A substituição da atmosfera real por uma atmosfera discreta e a aplicação de técnicas de análise numérica só poderão conduzir a resultados que aproximem a realidade observada, se previamente forem compreendidos os processos físicos e as consequências que as referidas técnicas implicam, as quais devem ser consistentes, convergentes e estáveis. A previsão numérica do tempo acabou por consolidar-se porque a teoria física, a teoria matemática e o desenvolvimento tecnológico na computação e nos sistemas de observação atingiram, em conjunto, um grau de maturidade suficiente, que garantiu o sucesso da modelação numérica da atmosfera. No entanto, há ainda muito caminho a percorrer, apesar dos avanços notáveis conseguidos nos últimos 50 anos.

Outra dificuldade decorrente da discretização, sempre presente, portanto inevitável, que foi reconhecida e controlada é o falseamento (aliasing), em consequência do qual a energia das perturbações de alta frequência é acumulada numericamente, logo de forma espúria, na energia das perturbações de baixa frequência, as quais influenciam directamente o tempo atmosférico. Não sendo o falseamento controlado, a não linearidade das equações conduz a uma instabilidade numérica, dita instabilidade não linear, que tem de ser evitada, alisando (smoothing) regularmente, no decurso da previsão, os diferentes campos meteorológicos.

Finalmente, há outro aspecto crucial a ter em consideração quando o continuum atmosférico é substituído por um discretum; com efeito, processos caracterizados por escalas inferiores à escala determinada pela discretização não podem ser resolvidos na rede ou grade que lhe corresponde. Tais processos designam-se por processos de subescala. Por exemplo, com uma malha horizontal de dimensão $100 \mathrm{~km}$ (ou $50 \mathrm{~km}$ ), processos como turbulência, atrito, convecção, transferência radiativa, efeitos dos aerossóis, efeitos das montanhas, transições de fase da água, microfísica de nuvens, etc. não têm representação directa no modelo considerado. Para uma dimensão de malha de $\Delta x$, a escala mais pequena que o modelo representa é $4 \Delta x$. No entanto, processos de subescala exercem, via interacções não lineares, influências significativas na escala sinóptica e na mesoscala, bem como na circulação geral da atmosfera. É pois imperativo incluir os efeitos dos processos de subescala, nas escalas superiores, em particular para períodos de previsão alargados. A referida inclusão faz-se parametrizando os efeitos estatísticos dos processos de subescala, i.e. exprimindo-os como funções dos campos que caracterizam as escalas resolvidas pelo modelo considerado. Aquelas funções passam a constituir termos fonte ou sumidouro nas equações, i.e. termos de geração ou destruição nas equações de prognóstico das variáveis associadas às escalas resolvidas. As formulações matemáticas e numéricas das diferentes parametrizações não são simples e têm também evoluído significativamente de há anos para cá, embora, em certos casos, estejam ainda na infância da arte, como é o caso da parametrização da convecção. 


\section{CONCLUSÃO}

Não se conhecem métodos para obter soluções das equações que regem o comportamento da atmosfera. A previsão do tempo faz-se com modelos numéricos que constituem uma representação mais ou menos elaborada da atmosfera real e dos processos que nela ocorrem.

Desde 1950, ano em que se realizou a primeira previsão de sucesso, utilizando o primeiro computador digital construído nos Estados Unidos da América, o ENIAC, a previsão numérica do tempo consolidou-se como ciência, graças aos avanços na compreensão de processos físicos e químicos que têm lugar na atmosfera, aos desenvolvimentos na análise numérica e ao vertiginoso progresso tecnológico, nos meios de computação e nos sistemas de observação da Terra.

A previsão do tempo continua a constituir um dos enormes desafios colocados à espécie humana. A sua evolução é o resultado da cooperação internacional, do saber científico, do desenvolvimento tecnológico, aspectos que verificando-se em simultâneo, ou com pequenos desfasamentos temporais, permitiram atingir o nível de desenvolvimento actual. Sem dúvida que a capacidade de prever o tempo tem contribuído para a preservação de vidas e bens e para a melhoria da qualidade de vida da humanidade.

\section{AGRADECIMENTO}

Este trabalho contou com o apoio técnico de Regina Corte-Real.

\section{BIBLIOGRAFIA}

Nebeker, F. (1995). Calculating the Weather, Meteorology in the 20th century, International
Geophysics Series (Vol. 60), Academic Press: London. 